\title{
TES Detector Noise Limited Readout Using SQUID Multiplexers
}

\author{
J.G. Staguhn ${ }^{1,3}$, D.J. Benford ${ }^{1}$, J.A. Chervenak ${ }^{1}$, , S.A. Khan ${ }^{1,4}$, \\ S.H. Moseley ${ }^{1}$, R.A. Shafer ${ }^{1}$, S. Deiker ${ }^{2}$, E.N.Grossman ${ }^{2}$, G.C. Hilton ${ }^{2}$, \\ K.D. Irwin ${ }^{2}$, C. Reintsema \\ ${ }^{1}$ NASA/Goddard Space Flight Center, Greenbelt, MD 20771, USA \\ ${ }^{2}$ NIST, Boulder, CO 80305, USA \\ ${ }^{3}$ SSAI, 5900 Princess Garden Parkway, Lanham, MD 20706, USA
}

\begin{abstract}
The availability of superconducting Transition Edge Sensors (TES) with large numbers of individual detector pixels requires multiplexers for efficient readout. The use of multiplexers reduces the number of wires needed between the cryogenic electronics and the room temperature electronics and cuts the number of required cryogenic amplifiers. We are using an 8 channel SQUID multiplexer to read out one-dimensional TES arrays which are used for submillimeter astronomical observations. We present results from test measurements which show that the low noise level of the SQUID multiplexers allows accurate measurements of the TES Johnson noise, and that in operation, the readout noise is dominated by the detector noise. Multiplexers for large number of channels require a large bandwidth for the multiplexed readout signal. We discuss the resulting implications for the noise performance of these multiplexers which will be used for the readout of two dimensional TES arrays in next generation instruments.
\end{abstract}

\section{SCIENTIFIC MOTIVATION}

Large arrays of fast, low temperature detectors are needed for a variety of disciplines, one of which is astronomy. The best suited wavelength range to study the early evolution of the universe and the assembly of the first prototype galaxies is the $\mathrm{FIR} / \mathrm{sub}-\mathrm{mm}$ regime. About half of the observable radiation energy in the universe is emitted in this wavelength range. The low photon energies combined with the requirement for large bandwidths and large arrays is still a challenge for detector development, since only bolometers meet these requirements. Semiconductor bolometers, which are the most commonly used devices at these wavelengths, cannot easily be multiplexed and are not well suited for high speed readout.

The Superconducting Transition Edge Sensor (TES) bolometer has been developed for use at wavelengths ranging from the sub-mm to X-rays. With its low impedance it is well matched for readout with SQUIDs. This scheme has a number of advantages, since SQUIDs can be operated at the detector temperature and are easily multiplexable. These properties, as well as the small time constants of TES devices, make it possible to develop large arrays of multiplexed TES detectors. 


\section{MULTIPLEXED READOUT OF DETECTOR ARRAYS}

\section{Large detector arrays require multiplexed readout}

For practical reasons, such as limitation of (cryogenic) wiring and amplifiers, large detector arrays require multiplexed readout. The fundamental properties of a multiplexing system are in principle identical, independent of the scheme of choice, be it frequency- or time domain multiplexing: the required bandwidth for the readout increases linearly with the number of pixels. The noise bandwidth of the multiplexed signal is larger than that of the signals from each individual pixel and the noise requirements for the readout system are correspondingly higher, unless the demultiplexed signal is appropriately filtered. For large arrays such filtering will be necessary and the methods to do so will differ, depending on the multiplexing scheme used.

\section{Multiplexer design}

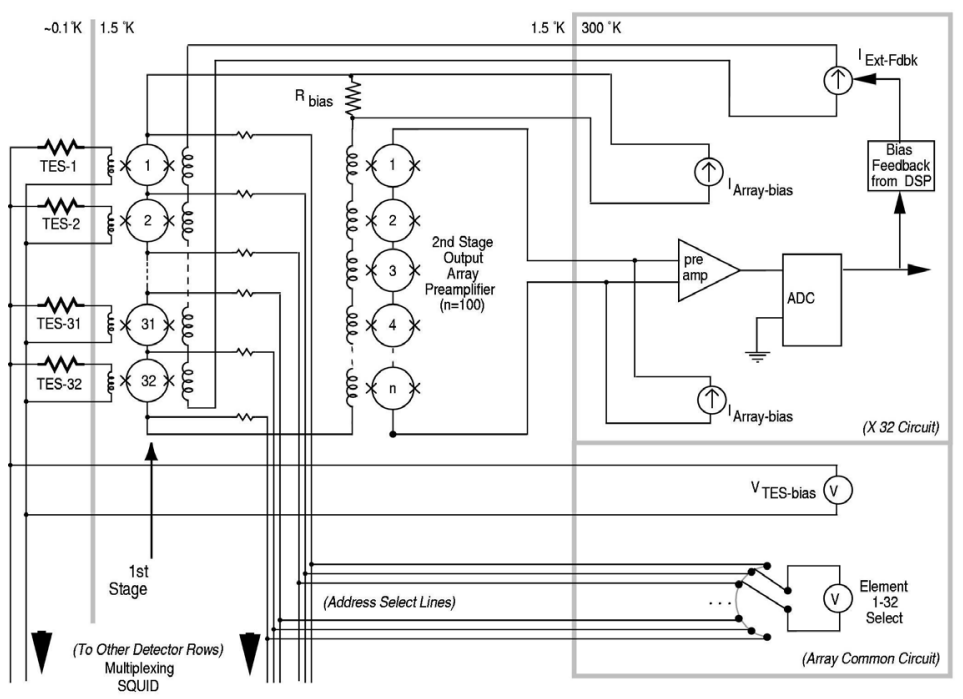

Figure 1. Schematics of the SQUID multiplexers

Figure 1 shows a schematic which illustrates the functionality of our NIST-designed SQUID multiplexers [1]. Each individual detector couples inductively into its own dedicated first stage SQUID. $n$ first stage SQUIDs for readout of the same number of detector pixels are stacked in series with $n+1$ electrical "address leads". By driving a bias current between one adjacent pair of address lines, only one SQUID will be operational while all other SQUIDs remain superconducting. Thus the output voltage over the entire series of first stage SQUIDs will be equal the voltage across the active SQUID. The TES is biased at all times and is low-pass filtered to a response time which is longer than the readout cycle of the entire first stage. 


\section{Noise performance measurements}

The phonon dominated white current noise density of TES is approximately given by $\mathrm{i}_{\mathrm{n}}=\sqrt{8 \mathrm{~kb} / \mathrm{R} \text { TES }}$. The Johnson current noise density of a general resistor $\mathrm{R}$ is given by: $\mathrm{i}_{\mathrm{J}}=\sqrt{4 \mathrm{k}_{\mathrm{b}} \mathrm{T} / \mathrm{R}}$. In order to verify the detector noise dominated performance of the multiplexer it is sufficient to perform Johnson noise measurements of resistors with comparable values of resistance. We tested 8 channel multiplexers which were cut from wavers that are one revision earlier than the SQUID chips we used for the first astronomical observations with multiplexed Transition Edge Sensor bolometers [2]. For the comprehensive noise performance tests we replaced the TES pixels with calibrated resistors. Figure 2 shows a multiplexed measurement of the Johnson noise of resistors at a temperature of $480 \mathrm{mK}$ and the corresponding theoretical predictions for the noise, including aliasing effects. The resistors were read out at a pixel frame rate of $10 \mathrm{kHz}$, yielding a bandwidth of $5 \mathrm{kHz}$ which is sufficient for the required detector speed. The multiplexed signal was bandpass limited to $100 \mathrm{kHz}$. Correspondingly all signals up to $100 \mathrm{kHz}$ are aliased into the $5 \mathrm{kHz}$ band (an approximation of the actual filter roll-off above $100 \mathrm{kHz}$ was incorporated in the calculations presented in this paper). The individual detector circuits contain low-pass filters. This allows the integration of the detector signal during one readout cycle. The individual low-pass filter device parameters and the model predictions for the noise are summarized in Table 1. All but one channel we measured are well modeled by a SQUID noise contribution of $4 \mathrm{pA} / \sqrt{\mathrm{Hz}}$. This value is consistent with the predicted SQUID multiplexer properties (the mutual inductance in our SQUID multiplexers is $500 \mathrm{pH}$ ). Only one channel (channel 5) has elevated SQUID noise, which is caused by a design error in the version of the multiplexers we used for the measurements. Figure 2 demonstrates the good agreement between theoretical predictions and measurements. If appropriately low-pass filtered, the Johnson noise of detectors operated at values higher than $100 \mathrm{~m} \Omega$ becomes comparable or even less (depending on filter rolloffs) than the aliased SQUID noise of our current eight channel multiplexer. In current ground based operations however we do not operate our detectors above $\sim 100 \mathrm{~m} \Omega$.

\section{Conclusion}

We have demonstrated the capability of our SQUID multiplexers to perform TES noise dominated readout. The mutual coupling between the SQUIDs and the detector circuits will be increased in the next generation of multiplexer chip designs leading to further improvements in the noise performance. Future large arrays with significant numbers of multiplexed pixels will nevertheless reach the limit at which the SQUID noise contribution is not negligible. In this case, however, fast sampling will make digital bandpass filtering possible. This will allow detector noise limited readout for a larger number of pixels, limited only by the sampling rate of the readout electronics. 


\section{8 channel multiplexed SQUID readout \\ $\mathrm{T}=480 \mathrm{mK}$}

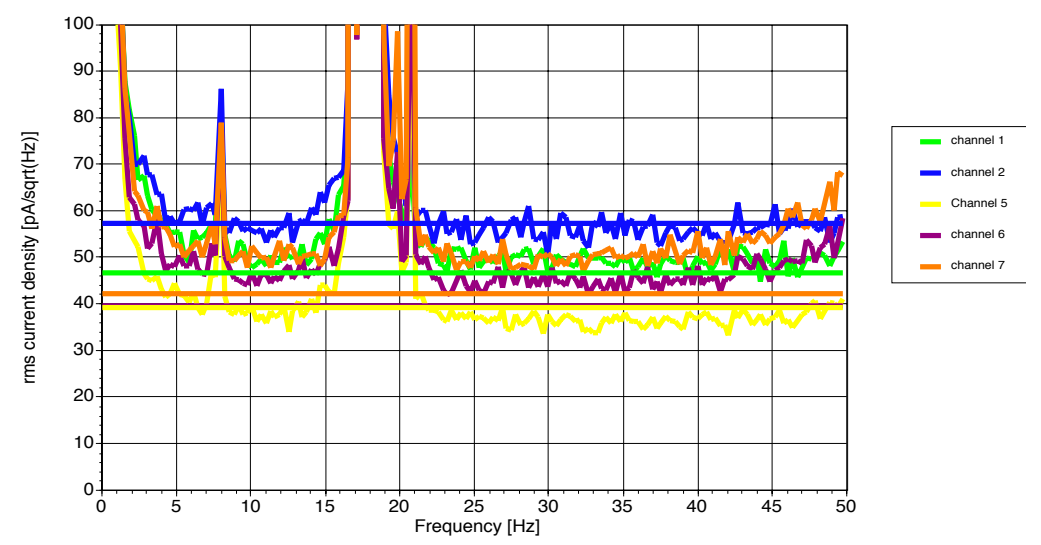

Figure 2. Multiplexed Johnson noise measurement with superimposed model predictions. The peak at $17 \mathrm{~Hz}$ corresponds to an acoustic resonance frequency of the wires in the dewar.

TABLE 1. Johnson noise density versus SQUID multiplexer noise density

\begin{tabular}{l|cccccc}
$\mathrm{T}=480 \mathrm{mK}$ & $\begin{array}{c}\mathrm{R} \\
{[\mathrm{m} \Omega]}\end{array}$ & $\begin{array}{c}\mathrm{R} / 2 \pi \mathrm{L} \\
{[\mathrm{kHz}]}\end{array}$ & $\begin{array}{c}\text { Johns. } \\
\text { Noise } \\
{[\mathrm{pA} / \sqrt{\mathrm{Hz}]}}\end{array}$ & $\begin{array}{c}\text { aliased } \\
\text { J.N. } \\
{[\mathrm{pA} / \sqrt{\mathrm{Hz}]}}\end{array}$ & $\begin{array}{c}\text { SQUID } \\
\text { Noise } \\
{[\mathrm{pA} / \sqrt{\mathrm{Hz}]}]}\end{array}$ & $\begin{array}{c}\text { aliased } \\
\text { SQUID n. } \\
{[\mathrm{pA} / \sqrt{\mathrm{Hz}}]}\end{array}$ \\
\hline Chan. 2 & 8 & 1.78 & 51.5 & 37.2 & 4.0 & 18.8 \\
Chan. 1 & 18 & 3.55 & 36.4 & 37.9 & 4.0 & 18.8 \\
Chan. 0 & 52 & 9.59 & 22.2 & 37.4 & 4.0 & 18.8 \\
Chan. 7 & 106 & 19.18 & 15.7 & 36.3 & 4.0 & 18.8 \\
Chan. 6 & 215 & 38.55 & 11.0 & 34.1 & 4.0 & 18.8. \\
Chan. 5 & Inf. & inf & 0.00 & 0.00 & 8.0 & 37.5
\end{tabular}

Note: Column 4 "aliased Johnson noise " shows the mean, low-pass filtered and aliased Johnson current noise density in the $5 \mathrm{kHz}$ band of the multiplexed channel readout. This value is less less than the nonlow-pass filtered Johnson noise of a resistor with the same resistance as channel 2. The low-pass filter device parameters are shown in column 2 . The theoretical values for channel 0 are displayed in the table even though it did not operate properly during the measurement and is not displayed in Fig. 1.

\section{REFERENCES}

1. Chervenak, J.A., Irwin, K.D., Grossmann, E.N., Martinis, J.M., Reintsema C.D, and Huber, M.E., Appl. Phys. Letters, 74 (26), 4043 (1999).

2. Staguhn, J.G. et al. "First Astronomical Use of Multiplexed Transition Edge Bolometers", these proceedings 